The other obvious danger of jumping onto the $\mathrm{PCMH}$ bandwagon without reformed payment models is that the intense amount of work that is done in care management or non-office-based care (ie, e-visits, phone visits) will go unrewarded. It is clearly in the interest of the insurance industry to encourage family physicians to fully embrace the PCMH model without having to pay for it. If we allow this to happen, we will doom ourselves to a practice model that is high demand but we will not be able to shrink our panel sizes or visit volumes to manageable levels and still keep our office open unless we are paid in a different way.

If we step back and look at what kind of payment model would best motivate physicians and their health care teams to perform at the highest level in the care of their patients, it would not be a fee-for-service model. The closer we tie the responsibility for the outcomes of care to both physician and patient, the greater the accountability. Developing primary care capitation payments to family medicine clinics based on population management with specific incentives for patient experience markers (a strong correlate to quality) and for key disease management and prevention measures would be our best blend of incentives for payment reform. Our European counterparts have experimented with multiple models and have found that having the bulk of a payment to physicians being a primary care capitation with careful incentives creates an optimal balance. The only way to resource clinics to carry out the work of an effective medical home is to shift more resources into the clinic via payment enhancements but how those payments are structured is critical to getting what we all wantaccessible, rational, quality primary care delivered by care teams led by family physicians.

How does this impact residency training? The simple answer is that if the $\mathrm{PCMH}$ is the model of care for now and the future, then we need to train residents in an environment that fulfills that model. However, given the high stress and high burnout risk, we need to couple our PCMH implementation with education on change management, burnout prevention, and leadership skills. In doing this we will position the next generation of family medicine graduates to be the $\mathrm{PCMH}$ leaders of the future.

Michael Tuggy, MD and Grant Hoekzema, MD;

Stoney Abercrombie, $M D_{i}$ Sneba Chacko, $M D_{i}$

Joseph Gravel, MD, Karen Hall, MD,

Lisa Maxwell, MD, Michael Mazzone, MD;

Todd Shaffer, MD, MBA, Martin Wieschbaus, MD

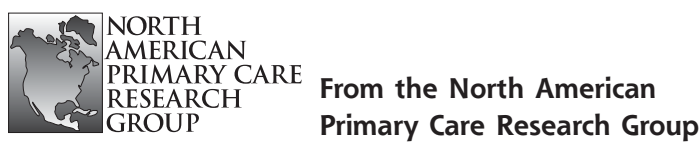

Ann Fam Med 2012;10:473-474. doi:10.1370/afm.1441.

\section{LARGE DATA SETS IN PRIMARY CARE RESEARCH}

With the widespread adoption of electronic health records (EHRs), researchers have growing access to large data sets that are being used for quality improvement, comparative effectiveness research, and public health policy decision making. In the recent past, large managed care organizations had almost exclusive access to these rich patient data sets. However, EHRs are rapidly leveling the playing field, with academic family medicine programs well positioned to take advantage of this resource and pioneer new fields of study. At the University of Wisconsin Department of Family Medicine (UW-DFM), we recently embarked on a study of polypharmacy that highlights the advantages and challenges of working with large EHR data sets and illustrates both what is possible and what the future may hold.

We began with a simple research question: "What are the patterns and predictors of medication use in our family medicine clinics?"1 Previous studies of polypharmacy have been limited to not only small sample sizes, but also focused primarily on elderly populations. Although insurance claims could provide us with a large, diverse sample, they generally do not include many clinically relevant over-the-counter medications and supplements. In addition, insurance claims do not capture prescription medications purchased without insurance, such as those on discount medication lists. Networked EHRs provide new opportunities for obtaining more comprehensive data regarding health services received, especially among populations who are discontinuously insured. ${ }^{2}$ Fortunately, UW-DFM has access to an EHR database from a network of 28 ambulatory-care clinics in Wisconsin that compiles over 300,000 annual visits. For the study described above, using anonymized data we were able to look at the prevalence of polypharmacy across a wide range of variables, including age, body mass index, smoking status, marital status, and major comorbidities. In the end, we analyzed nearly 2 million unique pieces of data from over 110,000 patients which, to our knowledge, far exceeds any previous study of polypharmacy.

Despite the readily available access to such vast data, our project highlights some of the challenges that face primary care researchers new to working 
with large EHR data sets. EHR data are gathered for the purposes of health care delivery, and as such, do not adhere to the rigorous standards of scientific studies. Although the sheer volume of data can overcome isolated inaccuracies, large systematic errors can occur. Our data, for example, contained several variables indicative of smoking status that frequently conflicted with one another. This necessitated looking at the entire data set for patterns of inconsistencies to ensure our findings were accurate. We also had to exercise caution not just with the available data, but with missing data as well. Missing data is a common issue with EHRs, and simply ignoring these gaps can lead to very biased results. We used several advanced statistical techniques to account for the uncertainly created by missing data in order to achieve appropriate confidence intervals. Ultimately, data exploration and cleaning constituted the majority of our efforts and should be a prime focus when analyzing EHR data. Finally, the issue of statistical significance takes on new meaning when working with thousands of data points. Unlike smaller studies, where considerable effort is expended to gather an adequate sample size, any sufficiently large data set will allow a researcher to find a "statistically significant" result. Consequently, large data sets require researchers to transition away from mechanistic statistical tests toward a mathematical modeling approach with the goal of discovering clinically relevant findings. ${ }^{3}$

After addressing these challenges, we were able to both arrive at an estimate of polypharmacy for a large, diverse adult population and identify some of the strongest predictors of heavy medication use. In doing so, we were able to look at segments of the population that were poorly studied and control for a wide range of variables. All of this was made possible by the use of a large EHR data set. We believe our exploratory study is merely scratching the surface of potential research that EHR data sets could ultimately provide. Academic family medicine programs are ideally situated to perform influential studies on population health, treatment effectiveness, disease prognosis, and social determinants of health. This research will not only enhance our understanding of disease, but shape how we practice medicine in the future. As a leader in disease management and preventive care, family medicine should capitalize on this new resource and lead the way in large dataset research.

Jon Meiman, $M D_{i}$ Jeff E. Freund, PharmD

\section{References}

1. Freund J, Meiman JG, Kraus C. Medication Use in a Network of Family Medicine Clinics. Poster session presented at: 45th Annual Spring Conference of the Society of Teachers of Family Medicine; Apr 25-29, 2012, Seattle Washington.
2. Devoe JE, Gold R, McIntire P, Puro J, Chauvie S, Gallia CA. Electronic health records vs Medicaid claims: completeness of diabetes preventive care data in community health centers. Ann Fam Med. 2011;9(4):351-358.

3. Rodgers JL. The epistemology of mathematical and statistical modeling: a quiet methodological revolution. Am Psychol. 2010;65(1):1-12.

\section{AAFP}

Ann Fam Med 2012;10:474-475. doi:10.1370/afm.1437.

\section{AAFP POSITION PAPER OPPOSES MANDATED CME, OTHER BARRIERS FOR PRESCRIBERS OF OPIOIDS}

In a position paper dated August 1, 2012, "Pain Management and Opioid Abuse," the American Academy of Family Medicine (AAFP) states that mandated CME could limit patient access to legitimate pain management needs. "Family physicians and other primary care clinicians play a vital role in effective pain management, including the prescribing of opioid analgesics. The creation of additional prescribing barriers for primary care physicians would limit patient access when there is a legitimate need for pain relief," the Academy said in a related news release.

"As such, the AAFP opposes any action that limits patients' access to physician-prescribed pharmaceuticals, and opposes any actions by pharmaceutical companies, public or private health insurers, legislation, the FDA or any other agency, which may have the effect of limiting by specialty the use of any pharmaceutical product."

These statements reiterate 2 existing AAFP policies, one of which opposes any action limiting patient access to physician-prescribed pharmaceuticals, and the other of which "opposes legislation or executive action that would require mandatory education of family physicians as a condition for prescribing specific drugs, such as opioids."

The Academy outlined several other major points in the paper, including its view that the chief goal of pain management should be to improve and maintain patients' ability to function. The AAFP also urged family physicians to individualize therapy based on review of the potential risks and benefits to each patient, possible drug side effects, and a functional assessment of the patient, and to monitor ongoing therapy accordingly.

In addition, the Academy:

- supports development of evidence-based physician education to ensure the safest and most effective 